\title{
Diferentes recipientes na produção de mudas de açaizeiro
}

\author{
Different containers on the production of açaizeiro seedllings \\ Diferentes contenedores en la producción de plántulas de açaizeiro
}

Recebido: 22/03/2021 | Revisado: 29/03/2021 | Aceito: 02/04/2021 | Publicado: 12/04/2021

\author{
Janaira Almeida Santos \\ ORCID: https://orcid.org/0000-0003-0788-5713 \\ Universidade do Estado do Pará, Brasil \\ E-mail: janairaalmeida14@gmail.com \\ Igor Vinicius de Oliveira \\ ORCID: https://orcid.org/0000-0003-4218-5587 \\ Universidade Federal do Sul e Sudeste do Pará, Brasil \\ E-mail: igor.oliveira@unifesspa.edu.br
}

\begin{abstract}
Resumo
$\mathrm{O}$ açaizeiro (Euterpe oleracea Mart.) é uma das principais culturas disseminadas na região amazônica com importância econômica, social e ambiental. O presente trabalho objetivou avaliar o uso de saco de polietileno e tubete com diferentes volumes na produção de mudas de açaizeiro. O ensaio foi conduzido no campo experimental da Universidade Federal do Sul e Sudeste do Pará entre abril e novembro de 2018, em Marabá, Pará. O delineamento experimental utilizado foi o inteiramente casualizado com 2 tratamentos e 4 repetições. Os recipientes avaliados foram sacos pretos perfurados com capacidade de $2800 \mathrm{~cm}^{3}$ e tubetes com capacidade de $265 \mathrm{~cm}^{3}$. A cultivar avaliada foi o BRS - Pará. Após germinação as plântulas foram transplantadas para os recipientes com substrato contendo $60 \%$ de areia, $20 \%$ de pó de serragem e $20 \%$ de esterco curtido. As avaliações foram feitas a cada 30 dias após o transplante realizando-se a contagem do número de folhas emitidas, a altura da planta e o diâmetro do coleto. A determinação de matéria seca total foi realizada aos 120 dias após o transplante. Os dados foram submetidos à análise de variância e as médias comparadas pelo teste de Tukey a 5\% e ao índice de qualidade de Dickson (IQD). Os parâmetros altura e massa seca total foram significativamente influenciados pelo volume do recipiente. $\mathrm{O}$ diâmetro do coleto e número de folhas das mudas produzidas nos sacos e nos tubetes não se diferenciaram estatisticamente. Mudas de açaí são melhor produzidas em sacos pretos perfurados com capacidade de $2800 \mathrm{~cm}^{3}$.
\end{abstract}

Palavras-chave: Viveiro; Sacos de polietileno; Tubetes.

\begin{abstract}
The açaizeiro (Euterpe oleracea Mart.) Is one of the main crops disseminated in the Amazon region with economic, social and environmental importance. The present work aimed to evaluate the use of polyethylene bag and tube with different volumes in the production of açaizeiro seedlings. The trial was conducted in the experimental field of the Federal University of the South and Southeast of Pará between April and November 2018, in Marabá, Pará. The experimental design used was a completely randomized design with 2 treatments and 4 repetitions. The evaluated containers were perforated black bags with a capacity of $2800 \mathrm{~cm}^{3}$ and tubes with a capacity of $265 \mathrm{~cm}^{3}$. The cultivar evaluated was BRS - Pará. After germination, the seedlings were transplanted into containers with substrate containing $60 \%$ sand, $20 \%$ sawdust and $20 \%$ tanned manure. The evaluations were made every 30 days after the transplant, counting the number of leaves emitted, the height of the plant and the diameter of the collect. The determination of total dry matter was carried out at 120 days after transplantation. The data were subjected to analysis of variance and the means compared by Tukey's test at 5\% and Dickson's quality index (IQD). The parameters height and total dry mass were significantly influenced by the volume of the container. The diameter of the collect and number of leaves of the seedlings produced in the bags and tubes did not differ statistically. Açaí seedlings are best produced in in perforated black bags with a capacity of $2800 \mathrm{~cm}^{3}$.
\end{abstract}

Keywords: Nursery; Polyethylene bags; Tubes.

\section{Resumen}

El açaizeiro (Euterpe oleracea Mart.) Es uno de los principales cultivos diseminados en la región amazónica con importancia económica, social y ambiental. Este trabajo tuvo como objetivo evaluar el uso de bolsas y tubos de polietileno con diferentes volúmenes en la producción de plántulas de açaizeiro. El ensayo se llevó a cabo en el campo experimental de la Universidad Federal del Sur y Sudeste de Pará entre abril y noviembre de 2018, en Marabá, Pará.El diseño experimental utilizado fue completamente al azar con 2 tratamientos y 4 repeticiones. Los envases evaluados fueron bolsas negras perforadas con una capacidad de $2800 \mathrm{~cm}^{3}$ y tubos con una capacidad de $265 \mathrm{~cm}^{3}$. El cultivar evaluado fue BRS - Pará, tras la germinación las plántulas se trasplantaron a recipientes con sustrato que contenía $60 \%$ arena, $20 \%$ aserrín y $20 \%$ estiércol curtido. Las evaluaciones se realizaron cada 30 días después del trasplante, contando el número de hojas emitidas, la altura de la planta y el diámetro del cuello. La determinación de materia seca 
total se realizó a los 120 días después del trasplante. Los datos se sometieron a análisis de varianza y se compararon las medias mediante la prueba de Tukey al 5\% y el índice de calidad de Dickson (IQD). Los parámetros altura y masa seca total fueron influenciados significativamente por el volumen del contenedor. El diámetro de la colección y el número de hojas de las plántulas producidas en las bolsas y tubos no difirieron estadísticamente. Las plántulas de açaî se producen mejor en bolsas negras perforadas con una capacidad de $2800 \mathrm{~cm}^{3}$.

Palabras clave: Guardería; Bolsas de polietileno; Tubos.

\section{Introdução}

O açaizeiro (Euterpe oleracea Mart.) é uma espécie frutífera que se destaca como uma das mais importantes na região amazônica, por possuir potencial agronômico, tecnológico, nutricional e econômico (Nogueira et al., 2005; Ximenes et al., 2020). Esse componente florestal nativo ocorre tanto em terras firmes como em várzeas sujeitas a inundações periódicas (Sousa \& Andrade, 2018), e está distribuído no baixo Amazonas, Maranhão, Tocantins e Amapá, alcançando as Guianas e a Venezuela (Souza, 1996). No Brasil há, pelo menos, dez espécies conhecidas de palmeiras do açaí, sendo duas delas as mais comuns na Amazônia: o açaizeiro de touceira (E. oleracea Mart.), espécie mais comumente encontrada nas várzeas da Amazônia Oriental, em especial nos estados do Pará, Amapá e Maranhão e o açaizeiro solteiro ou nativo da mata (E. precatória Mart.), encontrado tanto na terra firme como na várzea dos estados da Amazônia Ocidental (Amazonas, Rondônia, Acre) (Herraiz \& Ribeiro, 2013).

Ao longo dos últimos anos o mercado do açaí cresceu significativamente, ultrapassando as fronteiras amazônicas e se expandido para outras regiões do Brasil e do mundo, deixando de ser uma frutífera apenas regional (Oliveira et al., 2002; Coutinho, 2017). Parte desse crescimento está associado aos benefícios ligados à saúde, uma vez que a fruta possui altas concentrações de vitaminas, fibras, sais minerais e compostos com propriedades antioxidantes, tais como carotenoides e flavonoides, além de apresentar baixos níveis de calorias (Kang et al., 2011; Rufino, 2011; Veloso et al., 2015). De acordo com Cedrim et al. (2017), a importância do açaí como alimento funcional é por ele ser um alimento rico em antocianinas, que atuam modulando o metabolismo lipídico para melhora dos danos no organismo causados pelo estresse oxidativo, desencadeado por doenças crônicas, além de apresentar efeitos satisfatórios nos níveis de glicemia e pressão arterial, prevenindo e controlando efeitos da síndrome metabólica. O Estado do Pará é o maior produtor nacional de açaí com 151.793 toneladas de frutos extraídos anualmente (IBGE, 2021). O açaí faz parte da cultura alimentar paraense e constitui uma rica fonte de comercialização e de renda para os trabalhadores que se relacionam com esta atividade (Ribeiro, 2018).

Atualmente o açaizeiro se encontra entre as espécies mais promissoras e apresenta importância econômica com uma diversidade de produtos: polpa da fruta, artesanato, adubos, corantes, entre outros produtos; apresenta importância social, uma vez que representa valor cultural para as comunidades tradicionais; e importância ambiental, compondo a paisagem e a diversidade florística, servindo de alimento para a fauna e contribuindo na ciclagem de nutrientes (D'arace et al., 2019). Com uma cadeia produtiva que envolve extrativistas, produtores, intermediários, indústrias de beneficiamento e batedores artesanais, o açaí exerce importância crucial na formação de renda de expressivas famílias de pequenos produtores (Tavares et al, 2015).

No Pará, tanto a adoção de novas tecnologias no sistema de produção de açaizeiro quanto a expansão do consumo do fruto nos mercados nacional e internacional têm contribuído para a melhoria do bem-estar da população paraense, que vende e consome o fruto (Nogueira \& Santana, 2016). No entanto, ainda há necessidade de ampliar o programa de pesquisa visando à domesticação do gênero Euterpe, do processo de beneficiamento, novos produtos, mercado e normas legais ambientais, trabalhistas e previdenciárias (Tavares et al., 2017). Para Pias et al. (2015), a principal forma de alcançar a regularidade na produção e no mercado dessa cultura é promovendo atenção especial a todas as fases do seu ciclo produtivo, a começar pela sua formação inicial. A qualidade e o desenvolvimento das mudas, além dos fatores fisiológicos, possuem também alta correlação com o tipo de recipiente na qual esta foi plantada, pois as principais funções do recipiente são: conter o substrato 
que permite o crescimento e a nutrição das mudas; promover adequada formação do sistema radicular e proteger as raízes de danos mecânicos e desidratação e contribuir para a máxima sobrevivência e crescimento inicial no campo (Perin Neto, 2015; Zuffo et al., 2018).

A propagação do açaí pode ser feita de duas formas: assexuadamente através de perfilhos que aparecem na base da planta-mãe e sexuadamente através da semeadura das sementes em recipientes onde serão produzidas as mudas. Dentre os recipientes comumente utilizados, estão os sacos de polietileno, potes plásticos flexíveis e tubetes, que são encontrados em tamanhos variados (Carvalho \& Nascimento, 2018). Para o açaizeiro, a escolha do melhor recipiente deve levar em consideração o substrato utilizado e a idade desejada para a produção e/ou comercialização das mudas, visto que este exerce influência na sua qualidade e tem interferência direta em seu custo final, definindo a quantidade de substrato a ser utilizado, a mão-de-obra necessária no transporte, além de designar todo o manejo e a taxa de ocupação do viveiro, o tipo de sistema de irrigação e sua capacidade de produção anual (Queiroz et al., 2001).

Normalmente, os recipientes maiores são os que proporcionam melhor qualidade às mudas em geral, todavia, a utilização de recipientes com menores dimensões têm se mostrado efetivo na redução dos custos na fase de produção, transporte e distribuição em campo, proporcionando a formação de mudas de qualidade, com menor gasto e melhor rendimento na operação de plantio (Lima Filho et al., 2019). Apesar da cultura do açaizeiro ser amplamente disseminada na região, incipientes ainda são as pesquisas que apresentam resultados em relação ao cultivo racional e a recomendação das necessidades dessa cultura na fase de muda. Diante disso, ao se considerar a importância do açaizeiro no contexto agrícola paraense e a necessidade do aprofundamento de pesquisas visando estudos com recipientes de diferentes tamanhos e volumes, a pesquisa teve como objetivo avaliar o uso de saco de polietileno e tubete de diferentes volumes na produção de mudas de açaizeiro em viveiro.

\section{Metodologia}

O experimento foi conduzido em viveiro no campo experimental da Universidade Federal do Sul e Sudeste do Pará (5²1'54.6"S 4901'31.3"W) de Abril/2018 a Novembro/2018, no município de Marabá, estado do Pará. Segundo a classificação de Köppen o clima de Marabá é tropical semiúmido (Aw) com temperaturas que variam ao longo do ano entre 22 ${ }^{\circ} \mathrm{C}$ a $35{ }^{\circ} \mathrm{C}$, e volume médio de precipitação elevado, próximo aos $2.200 \mathrm{~mm}$ anuais (Inmet, 2018; Medeiros et al., 2020). O viveiro utilizado foi do tipo telado coberto com sombrite de 50\%. O delineamento experimental utilizado foi o inteiramente casualizado com 2 tratamentos e 4 repetições. Com intuito de garantir a uniformidade do ensaio foram obtidas, através de doação do Instituto de Desenvolvimento Florestal e da Biodiversidade do Estado do Pará - Ideflor-Bio, sementes pré germinadas da cultivar BRS - Pará. Nessa etapa realizada pelo Ideflor-Bio, as sementes foram acondicionadas em sacos de plástico transparente fechados, misturadas com areia e pó de serragem na proporção 1:1 (Farias Neto et al., 2010).

Após emergência das plântulas, estas foram transplantadas para os recipientes em substrato com areia, pó de serragem e esterco curtido na proporção de 3:1:1 de acordo com a metodologia recomendada por Bentes - Gama et al. (2005). As principais características químicas do substrato estão apresentadas na Tabela 1.

Tabela 1: Principais propriedades químicas do substrato utilizado no experimento.

\begin{tabular}{|c|c|c|c|c|c|c|c|c|c|}
\hline $\mathrm{pH}$ & $\mathrm{Ca}$ & $\mathrm{Mg}$ & $\mathrm{Ca}+\mathrm{Mg}$ & $\mathrm{H}+\mathrm{Al}$ & $\mathrm{P}$ & $\mathrm{K}$ & Argila & Mat. Org & CTC \\
\hline \multicolumn{5}{|c|}{$\mathrm{cmolc} \mathrm{kg}^{-1}$} & \multicolumn{2}{|c|}{$-\mathrm{mg} \mathrm{kg}^{-1}-$} & \multicolumn{2}{|c|}{------g kg-1------ } & cmolc kg $^{-1}$ \\
\hline 6,8 & 9,3 & 4,2 & 13,5 & 1,1 & 9 & 156 & 60,0 & 58,0 & 15,0 \\
\hline
\end{tabular}

Fonte: Autores. 
Para avaliação do melhor recipiente foram utilizados sacos pretos perfurados de polietileno nas dimensões de 12 x 25 com capacidade de $2800 \mathrm{~cm}^{3}$ e tubetes com capacidade de $265 \mathrm{~cm}^{3}$ acondicionados em bandejas próprias. Cada parcela constou de 12 sacos e 12 tubetes totalizando 96 mudas. Os tratamentos foram dispostos em casa de vegetação de forma intercalada, com tubetes distribuídos em bandejas e os sacos de plástico sobre terreno nivelado. A irrigação foi realizada em sistema de aspersão todos os dias pela manhã e pela tarde por 40 minutos. O controle de plantas espontâneas foi realizado sempre que necessário pelo método de capina manual. Durante o período de avaliação foi realizada aplicação de calda de pimenta do reino, alho e sabão (Costa \& Campanhola, 1997) para prevenção de pulgão preto (Cerataphis lataniae Boisuval), importante praga do açaizeiro (Souza \& Lemos, 2004).

As avaliações foram feitas a cada 30 dias após o transplantio realizando-se a contagem do número de folhas fotossinteticamente ativas, a altura da planta até a inserção da última folha totalmente aberta e o diâmetro do coleto, medido a 1 $\mathrm{cm}$ do substrato com auxílio de paquímetro digital $(0,01 \mathrm{~mm})$. A determinação de matéria seca foi realizada aos 120 dias após o transplante, as mudas foram acondicionadas em estufas com circulação forçada de ar, a $60^{\circ} \mathrm{C}$, até peso constante, para a determinação do peso da matéria seca da parte aérea e do sistema radicular.

Para efeito comparativo e a partir dos dados obtidos para os parâmetros de diâmetro do coleto, de altura da planta e da biomassa seca das mudas, calculou-se o Índice de Qualidade de Dickson (IQD) utilizando-se o software Microsoft Excel através da Equação 1 (Dickson et al., 1960).

$$
\mathrm{IQD}=\operatorname{MST}(\mathrm{g})[\mathrm{H}(\mathrm{cm}) / \mathrm{DC}(\mathrm{mm})]+[\mathrm{MSPA}(\mathrm{g}) / \mathrm{MSR}(\mathrm{g})]
$$

Em que:

MST = Massa Seca Total;

$\mathrm{H}=$ Altura da Planta;

DC = Diâmetro do Coleto;

MSPA = Massa Seca da Parte Aérea; e

MSR = Massa Seca da Raiz.

Os resultados foram submetidos a análise de variância aplicando-se o Teste F e de Tukey a 5\% utilizando-se o software Sistema para Análise de Variância - Sisvar (Ferreira, 2011).

\section{Resultados e Discussão}

De acordo com os resultados apresentados na Tabela 2, pode-se observar que aos 120 dias após a repicagem os recipientes testados influenciaram as variáveis altura da planta (FP) e massa seca total (MST) $(\mathrm{P}<0,05)$.

Tabela 2: Resumo da análise de variância das médias do diâmetro do coleto (DC), altura da planta (H), número de folhas (FP) e massa seca total (MST) das mudas de açaizeiro no recipiente saco e tubete.

\begin{tabular}{l|c|c|c|c}
\hline \multicolumn{1}{c|}{ FV } & DC & H & FP & MST \\
\hline \hline Saco & $5,23^{\mathrm{a}}$ & $24,30^{\mathrm{a}}$ & $2,33^{\mathrm{a}}$ & $1,13^{\mathrm{a}}$ \\
\hline Tubete & $4,58^{\mathrm{a}}$ & $20,32^{\mathrm{b}}$ & $2,25^{\mathrm{a}}$ & $0,83^{\mathrm{b}}$ \\
\hline C.V. (\%) & 0,09 & 0,12 & 0,02 & 0,21 \\
\hline
\end{tabular}

Nota: Médias seguidas de mesma letra não diferem pelo teste de Tukey (P>0,05).

Fonte: Autores. 
Em relação ao diâmetro do coleto (DC) e o número de folhas (FP), não houveram diferenças significativas entre as mudas de açaizeiro produzidas nos sacos plásticos e as dos tubetes, indicando que as mudas responderam positivamente ao cultivo nos recipientes com diferentes volumes até os 120 dias. Tanto o DC quanto o FP tem sido considerado um bom indicativo do vigor das mudas e dos índices de produtividade, pois refletem no desempenho das plantas em campo e em menores taxas de mortalidade (Colder \& Saldanha, 2006; Mendes et al., 2018). Nas variáveis altura das plantas (H) e massa seca total (MST), as mudas produzidas em saco plástico apresentaram valores médios significativamente maiores que as do tubete, revelando que o volume do recipiente está diretamente relacionado com o maior desempenho desses parâmetros. Resultados semelhantes foram observados por Torres (2019) e Nascimento e Gatti (2019) em que o melhor padrão morfológico de mudas de açaizeiro foi decorrente de cultivo em sacos de polietileno de $1000 \mathrm{~cm}^{3}$ e tubetes com volume de $280 \mathrm{~cm}^{3}$.

Esse comportamento de melhor desenvolvimento das mudas produzidas em sacos plásticos possivelmente está relacionado com o maior volume de substrato disposto nesse tipo de recipiente, pois, apesar de reduzirem a necessidade de área do viveiro e os custos de produção, os recipientes menores podem restringir o crescimento do sistema radicular das mudas de diferentes espécies (Lima et al., 2006; Malavasi, 2006; Lopes, 2017; Melo et al., 2018) que, consequentemente, tendem a apresentar um crescimento balanceado comprometendo o seu estabelecimento no campo. Uma característica que também deve ser considerada é o sistema radicular fasciculado do açaizeiro (Henderson \& Galeano, 1996). Sistemas radiculares desse tipo não possuem uma raiz principal, diante disso, o volume de substrato no tubetes provavelmente foi restritivo para o crescimento potencial das raízes e consequentemente exerceu influência no parâmetro de altura e no percentual de MST.

Outro fator que pode ter influenciado nesse resultado está relacionado ao manejo da irrigação em recipientes pequenos como os tubetes. Esses tipos de recipientes podem demandar maior frequência de irrigação para que o substrato mantenha umidade, uma vez que o açaizeiro é exigente em água (Viana et al., 2018). Gervásio (2003) chama atenção para o fato de que particularidades nesse sentido associado a outros fatores implicam as mudas o risco de estresse hídrico que deve ser evitado para se conseguir teor de umidade no substrato e evitar o acúmulo de sais. A falta de umidade no substrato pode tornar as plantas vulneráveis a pragas e doenças, além de induzir o fechamento estomático, reduzir o crescimento, causar o acúmulo de solutos e antioxidantes, reduzir a área foliar e o crescimento do caule (Costa et al., 2017; Rocha et al., 2017).

No Pará, a Comissão Estadual de Sementes e Mudas do Pará (Cesm-Pa) estabelece normas e padrões onde as mudas de açaizeiro, para serem comercializadas, deverão apresentar, no mínimo, cinco folhas fisiologicamente ativas, com diâmetro do coleto maior que a do ápice das mudas, altura entre 40 a $60 \mathrm{~cm}$ medida a partir do coleto, apresentar sistema radicular bem desenvolvido e ter de quatro a oito meses de idade a partir da emergência das plântulas. Essas características não puderam ser observadas neste trabalho em virtude do tempo de desenvolvimento no qual as mudas foram avaliadas.

\section{1 Índice de qualidade das mudas}

As mudas produzidas em saco plástico apresentaram valores médios de índice de qualidade de Dickson (IQD) superiores aos do tubete (Figura 1). 
Figura 1: Índice de Qualidade de Dickson de mudas de açaizeiro produzidas em saco e tubete.

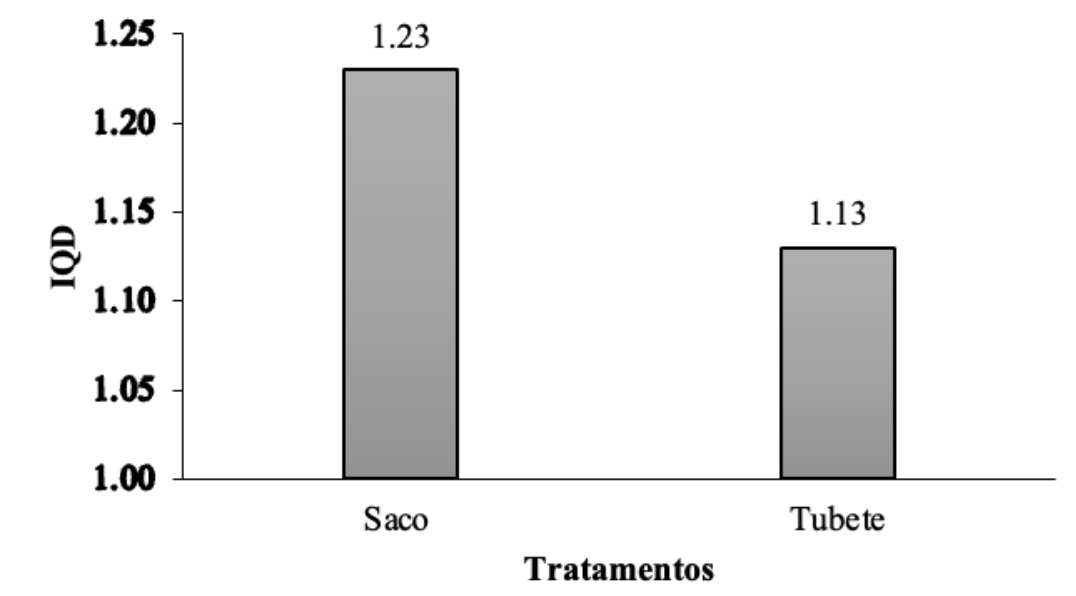

Fonte: Autores.

O maior IQD obtido após 120 dias foi de 1,23, tais valores são inferiores aos que Pereira (2017) obteve ao avaliar o desenvolvimento vegetativo de mudas de açaizeiro cultivada em três tamanhos de recipientes $(20 \times 30 \mathrm{~cm}, 17$ x $20 \mathrm{~cm}, 11 \times 12$ $\mathrm{cm}$ ), onde o IQD foi de 4,94 aos 180 dias. O índice de qualidade de Dickson (IQD) é considerado uma medida que integra características morfológicas, o que possibilita verificar a distribuição da biomassa, além de ser uma ferramenta importante para avaliar se as mudas estão aptas para plantio em local definitivo, uma vez que engloba vários parâmetros indicadores de sobrevivência e de qualidade (Fonseca et al., 2002; Bonamigo et al., 2016). Para o açaizeiro o que dificulta a análise dos índices é a ausência de uma recomendação de IQD para a espécie. Entretanto, quanto maior é o valor gerado pelo índice, melhor é o estado da muda e maiores as chances de êxito do desempenho das mudas de várias espécies em campo (Gomes, 2003; Rudek et al., 2013). Para correlação com o teste F e o teste de Tukey ( $p<0,05)$ os resultados obtidos através do índice de qualidade das mudas produzidas nesse ensaio foram satisfatórios e seguiram padrões semelhantes.

\section{Conclusão}

O desenvolvimento de mudas de açaizeiro é influenciado pelo volume dos recipientes, uma vez que tubetes com capacidade de $265 \mathrm{~cm}^{3}$ limitaram o crescimento das mudas nos parâmetros altura das plantas e massa seca total. Os parâmetros diâmetro do coleto e número de folhas responderam positivamente ao cultivo tanto nos sacos plásticos quanto nos tubetes, e não se diferenciaram estatisticamente. Mudas de açaí são melhor produzidas até os 120 dias em sacos pretos perfurados com capacidade de $2800 \mathrm{~cm}^{3}$. De acordo com o índice de qualidade de Dickson, as mudas produzidas em sacos plásticos obtiveram maior índice $(1,23)$ quanto as mudas produzidas nos tubetes $(1,13)$.

\section{Referências}

Bentes - Gama, M. M., Ribeiro, G. D., Fernandes, C. D. F., \& Medeiros, I. D. (2005). Açaí (Euterpe spp.): características, formação de mudas e plantio para a produção de frutos. Circular Técnica, Embrapa-RO. Porto Velho. https://www.embrapa.br/busca-de-publicacoes/-/publicacao/859446/acai-euterpe-sppcaracteristicas-formacao-de-mudas-e-plantio-para-a-producao-de-frutos

Bonamigo, T., Scalon, S. de P. Q., \& Pereira, Z. V. (2016). Substratos e níveis de luminosidade no crescimento inicial de mudas de Tocoyena formosa (Cham. \& Schltdl.) K. Schum. (RUBIACEAE). Ciência Florestal, 26(2), 501-511. http://dx.doi.org/10.5902/1980509822750 
Cedrim, P. C., Silva, A. B., Albuquerque, E. M., \& Nascimento, T. G. do. (2018). Propriedades antioxidantes do açaí (Euterpe oleracea) na síndrome metabólica. Brazilian Journal of Food Technology, 21, e2017092. https://doi.org/10.1590/1981-6723.09217

Colder, M. P. M., \& Saldanha, C. W. (2006). Germinação de sementes e crescimento de plântulas de diferentes progênies de Euterpe edulis Mart. Revista Árvore, 30(5), 693-699. https://doi.org/10.1590/S0100-67622006000500002

Costa, J. C. F. da, Mendonça, R. M. N. M., Fernandes, L. F., Oliveira, F. P. de, \& Santos, D. (2017). Caracterização física de substratos orgânicos para o enraizamento de estacas de goiabeira. Revista Brasileira De Agropecuária Sustentável, 7(2). https://doi.org/10.21206/rbas.v7i2.390

Costa, M. D., \& Campanhola, C. (1997). A agricultura alternativa no Estado de São Paulo. Jaguariúna: Embrapa-CNPMA.

Coutinho, R. V. (2017). A exploração do açaí como alternativa para o desenvolvimento econômico da Amazônia Legal: estudo de caso do Estado do Pará (1990-2010). Dissertação de Mestrado, Universidade Federal de Roraima, RR, Brasil. http://repositorio.ufrr.br:8080/jspui/handle/prefix/147

D'arace, L. M. B., Pinheiro, K. A. O., Gomes, J. M., Carneira, F. S., Costa, N. S. L., Rocha, E. S., \& Santos, M. L. (2019). Produção de açaí na região norte do Brasil. Revista Ibero Americana de Ciências Ambientais, 10(5), 15-21. http://www.sustenere.co/index.php/rica/article/view/CBPC2179-6858.2019.005.0002

Dickson, A., Folha, A. L., \& Hosner, J. F. (1960). Quality appraisal of white spruce and white pine seedling stock in nurseries. Forest Chronicle, 36, p.10-13.

Farias Neto, J. T., Vasconcelos, M. A. M., \& da Silva, F. C. F. (2010). Cultivo, processamento, padronização e comercialização do açaí na Amazônia. https://www.agencia.cnptia.embrapa.br/Repositorio/Frutal+-+2010-1_000gbtedrex02wx5ok07shnq9qr3osfo.pdf

Ferreira, D. F. (2011). Sisvar: a computer statistical analysis system. Ciência e Agrotecnologia, 35(6), 1039-1042. https://dx.doi.org/10.1590/S141370542011000600001

Fonseca E. P. (2002). Padrão de qualidade de mudas de Trema micrantha (L.) Blume, produzidas sob diferentes períodos de sombreamento. Revista Árvore, 26(4), 515-523. http://dx.doi.org/10.1590/S0100-67622002000400015.

Gervásio, E. S. (2003). Efeito de lâminas de irrigação e doses de condicionador, associadas a diferentes tamanhos de tubetes, na produção de mudas de cafeeiro. Tese (Doutorado em Agronomia) - Escola Superior de Agricultura Luiz de Queiroz, Piracicaba, SP, Brasil. https://www.teses.usp.br/teses/disponiveis/11/11143/tde-23092003-135210/publico/eliezer.pdf

Gomes, J. M., Couto, L., Leite, H. G., Xavier, A., \& Garcia, S. L. R. (2003). Crescimento de mudas de Eucalyptus grandis em diferentes tamanhos de tubetes e fertilização NPK. Revista Árvore, 27(2), 113-127.

Henderson, A., \& Galeano, G. (1996). Euterpe, Prestoea, and Neonicholsonia (Palmae: Euterpeinae). New York: New York Botanical Garden, 90p.

Herraiz, A. D., \& Ribeiro, P. N. T. (2013). Manejo e cultivo de açaí na calha do rio Madeira, Sul do Amazonas. Projeto Fronteiras Florestais (p.1-48). Humaitá, AM.

Instituto Brasileiro de Geografia e Estatística (2021). Produção vegetal nacional. https://sidra.ibge.gov.br/tabela/289\#resultado

Instituto Nacional De Meteorologia (2018). Marabá. http://sisdagro.inmet.gov.br/sisdagro/app/climatologia/bhclimatologicomensal/index. Acesso em: 10 de março de 2018

Kang, J., Xie, C., Li, Z., Nagarajan, S., Schauss, AG, Wu, T., \& Wu, X. (2011). Flavonóides da polpa do açaí (Euterpe oleracea Mart.) E suas atividades antioxidantes e antiinflamatórias. Food Chemistry, 128 (1), 152-157.

Lima Filho, P., Leles, P. S. dos S., Abreu, A. H. M. de, Silva, E. V. da, \& Fonseca, A. C. da. (2019). Produção de mudas de Ceiba speciosa em diferentes volumes de tubetes utilizando o biossólido como substrato. Ciência Florestal, 29(1), 27-39. https://doi.org/10.5902/1980509819340

Lima, R. L. S., Soares, L. S., Silva, M. I. L., Vale, L. S., \& Beltrão, N. E. M. (2006). Volume de recipientes e composição de substratos para produção de mudas de mamoneira. Ciência e Agrotecnologia, 30(3), 480-486. https://doi.org/10.1590/S1413-70542006000300014

Lopes, F. A. (2017). Desenvolvimento de mudas de macaúba em função do tamanho do recipiente e idade da muda na fase de viveiro. Dissertação de Mestrado, Universidade Federal de Viçosa, MG, Brasil. https://www.locus.ufv.br/handle/123456789/25204

Malavasi, U. C., \& Malavasi, M. M. (2006). Efeito do volume do tubete no crescimento inicial de plântulas de Cordia trichotoma (Vell.) Arrab. ex Steud e Jacaranda micranta Cham. Ciência Florestal, 16(1), 11-16. https://doi.org/10.5902/198050981883

Medeiros, N. L., Junior, A. P., dos Santos Jesus, E., \& Jesus, N. D. V. G. Variabilidade espacial do conforto térmico em pontos distintos do município de Marabá-pa. Ciências ambientais: climatologia, geotecnologias, mineração e estudos de monitoramento/Altem Nascimento Pontes; Alessandro Silva do Rosário (Orgs.). Belém: EDUEPA, 2020. 166 p.: il. Inclui bibliografias, 87.

Melo, L. A. de, Abreu, A. H. M., Leles, P. S. S., Oliveira, R. R., \& Silva, D.T. (2018). Qualidade e crescimento inicial de mudas de Mimosa caesalpiniifolia Benth. produzidas em diferentes volumes de recipientes. Ciência Florestal, 28(1), 47-55. https://www.scielo.br/scielo.php?script=sci_arttext\&pid=S198050982018000100047

Mendes, N. V. B., Lima, D. C., Corrêa, M. C. M., \& Natale, W. (2018). Emergência e desenvolvimento inicial do açaizeiro em diferentes substratos e ambientes. Acta Iguazu, 7(2), 84-96. http://e-revista.unioeste.br/index.php/actaiguazu/article/view/16287/12769

Nascimento, W. M. O., \& Gatti, L. A. P. (2019). Recipientes para produção de mudas de Euterpe oleracea. In Embrapa Amazônia Oriental-Artigo em anais de congresso (ALICE). In: Congresso brasileiro de fruticultura, 26., 2019, Juazeiro, Petrolina. Anais... Juazeiro, Petrolina: Sociedade Brasileira de Fruticultura.https://www.alice.cnptia.embrapa.br/bitstream/doc/1115144/1/AnaisCBF2019final885888.pdf

Nogueira, A. K. M., \& Santana, A. C. de S. (2016). Benefícios socioeconômicos da adoção de novas tecnologias no cultivo do açaí no Estado do Pará. Revista Ceres, 63(1), 001-007. http://www.ceres.ufv.br/ojs/index.php/ceres/article/view/222 
Nogueira, O. L., Figueirêdo, F. J.C., \& Muller, A. A. (2005). Sistemas de produção de açaí. Belém, PA. Embrapa Amazônia Oriental. 1. Ed. http://ainfo.cnptia.embrapa.br/digital/bitstream/item/125409/1/SISTEMA-PROD-4-ONLINE-.pdf

Oliveira, M. do S. P. de, Carvalho, J. E. U., Nascimento, W. M. O., \& Müller, C. H. (2002). Cultivo do Açaizeiro para Produção de Frutos. Circular Técnico, Belém, PA. https://www.agencia.cnptia.embrapa.br/Repositorio/Producaodefrutos+Circ_tec_26_000gbz56rpu02wx5ok01dx91cobm2bes.pdf

Pereira, T. R. S. (2017). Desenvolvimento vegetativo de Euterpe Oleraceae cultivada em diferentes tamanhos de recipientes e proporções de substratos. Monografia, Universidade Federal do Recôncavo da Bahia - UFRB. Cruz das Almas, BA, Brasil. http://www.repositoriodigital.ufrb.edu.br/bitstream/123456789/1220/1/TCC_Thaise_vers\%C3\%A3odigital.pdf

Pias, O. H. C. de, Berghetti, J. L. S., \& Cantarelli, E. B. (2015). Qualidade de mudas de cedro em função da utilização de fertilizantes e recipientes de diferentes tamanhos. Revista Agro@mbiente, 9(2), 208-213. https://revista.ufrr.br/agroambiente/article/viewFile/2210/1586>

Pierin Neto, L. (2015). Influência do recipiente na qualidade de mudas de Euterpe edulis Martius (juçara) e Araucaria angustifolia (Bertol.) Kuntze (araucária). https://acervodigital.ufpr.br/bitstream/handle/1884/44534/LEONE\%20PIERIN\%20NETO.pdf?sequence=1. Acesso em: 02 de abril de 2018.

Queiroz, J. A. L. de, \& Mochiutti, S. (2001). Cultivo de açaizeiros e manejo de açaizais para produção de frutos. Macapá: Embrapa Amapá, 34 p. (Embrapa Amapá. Documentos, 30). https://www.embrapa.br/busca-de-publicacoes/-/publicacao/346787/cultivo-de-acaizeiros-e-manejo-de-acaizais-para-producao-defrutos

Ribeiro, F. (2018). Trabalhadores do açaí: história, memória e ambiente. Trabalhadores, migrações e natureza no brasil equatorial, 89. https://www2.unifap.br/editora/files/2018/05/Livro-Trabalhadores-Migra\%c3\%a7\%c3\%b5es-e-Natureza-no-Brasil-Equatorial.pdf

Rocha, C. W., Reis, M. A., Silva, M. A., Saraiva, T. A., \& Dayrell, D. M. (2017). Uso de Diferentes Substratos na Produção de Mudas de Maracujazeiro Amarelo. Revista Agroveterinária, Negócios e Tecnologias, 2(1), 38-51. http://ojs.fccvirtual.com.br/index.php/REVISTA-AGRO/article/view/11

Rudek, A., Garcia, F. A. O., \& Bandeira, F. S. (2013). Avalição da qualidade de mudas de eucalipto pela mensuração da área foliar com o uso de imagens digitais. Enciclopédia Biosfera, $37(17), \quad 375-$ https://www.conhecer.org.br/enciclop/2013b/CIENCIAS\%20AGRARIAS/avaliacao\%20da\%20qualidadede\%20mudas.pdf

Rufino, M. S. M. (2011). Açaí (Euterpe oleraceae) 'BRS Pará': A tropical fruit source of antioxidant dietary fiber and high antioxidant capacity oil. Food Research International, 44(7), 2100-2106.

Sousa, P. C. de, \& Andrade, V. M. S. (2018). Potencialidade na produção de Euterpe oleracea Mart (Açaí) em comunidades tradicionais na Amazônia. Revista Contribuciones a las Ciencias Sociales, (1), 1. https://www.eumed.net/rev/cccss/2018/01/produccion-euterpe-amazonia.html

Souza, A. G. C., Sousa, N. R., Silva, S. E. L.; Nunes, C. D. M., Canto, A. C., \& Cruz, L. A. A. (1996). Fruteiras da Amazônia. Brasília, DF: Embrapa-SPI; Manaus: Embrapa-CPAA. https://www.infoteca.cnptia.embrapa.br/infoteca/handle/doc/664664

Souza, L. A., \& Lemos, W. D. P. (2004). Prospecção de insetos associados ao açaizeiro (Euterpe oleracea Mart.) em viveiro e proposições de controle. Embrapa Amazônia Oriental-Artigo em (ALICE). https://www.alice.cnptia.embrapa.br/alice/bitstream/doc/577381/1/revistadecienciasagrarias42231241.pdf

Tavares, G. D. S., \& Homma, A. K. O. (2015). Comercialização do açaí no estado do Pará: alguns comentários. Embrapa Amazônia Oriental-Artigo em periódico indexado (ALICE). https://www.eumed.net/cursecon/ecolat/br/15/acai-para.html

Tavares, G. D. S., Homma, A. K. O., \& de Menezes, A. J. E. A. (2017). Comercialização de polpa de açaí no estado do Pará. In Embrapa Amazônia OrientalArtigo em anais de congresso (ALICE). In: Simpósio sober norte, 1., 2017, Belém, PA. Anais... Belém, PA: Sober norte, 2017. https://www.alice.cnptia.embrapa.br/handle/doc/1074306

Torres, C. M. (2019). Qualidade morfofisiológica de mudas de Euterpe oleracea (Mart.) produzidas em recipientes de diferentes volumes. Dissertação de Mestrado, Universidade Federal Rural da Amazônia, Belém, PA, Brasil. http://repositorio.ufra.edu.br/jspui/handle/123456789/673

Veloso, C. A. C., Silva, A. R., Carvalho, E., Sales, A., das Chagas, C. T. G., \& Freire, A. (2015). Influência da adubação NPK na formação do açaizeiro em Latossolo Amarelo do Nordeste Paraense. In Embrapa Amazônia Oriental-Artigo em anais de congresso (ALICE). In: Congresso brasileiro de ciência do solo, 35., 2015, Natal. O solo e suas múltiplas funções: anais. Natal: Sociedade Brasileira de Ciência do Solo, 2015.

Viana, L. F. (2018). Análise Econômica do Cultivo de Açaizeiro (Euterpe oleracea Mart.) Irrigado no Nordeste Paraense: um estudo de caso no município de Igarapé-Açu, PA. Dissertação de Mestrado, Universidade do Bará, Belém. https://paginas.uepa.br/pcambientais/dissertacao_laisa_viana_turma_2016.pdf

Ximenes, L. C., Gama, J. R. V., Bezerra, T. G., \& Cruz, G. S. (2020). Importância do açaí na renda mensal da comunidade quilombola Murumuru em Santarém, Pará. Revista Ibero Americana de Ciências Ambientais, 11(2), 36-42. http://sustenere.co/index.php/rica/article/view/3683

Zuffo, A. M., Steiner, F., Bush, A., Zuffo Júnior, J. M., \& Santos, D. M. S. (2018). Tamanho de recipientes na formação de mudas de Peltophorum dubium (Sprengel) Taubert (Fabaceae). Engenharia na Agricultura, 26(3), 258-268. https://periodicos.ufv.br/reveng/article/view/751 